Chirurg

DOI 10.1007/s00104-017-0448-6

(c) Der/die Autor(en) 2017. Dieser Artikel ist eine Open-Access-Publikation.

CrossMark

M. J. Pollheimer $\cdot$ C. Langner

Institut für Pathologie, Medizinische Universität Graz, Graz, Österreich

\title{
Pathologie der R1-Klassifikation in der viszeralonkologischen Chirurgie
}

Die TNM(Tumor/Nodus/Metastasen)Klassifikation ist das weltweit am häufigsten angewandte System zur Beschreibung der anatomischen Ausbreitung maligner Tumoren. In dieser Übersicht wird am Beispiel verschiedener viszeralonkologischer Tumoren speziell auf die Resektionsrandanalyse eingegangen.

\section{Die R-Klassifikation}

Die Residualtumorklassifikation (RKlassifikation) stellt eine zusätzliche Klassifikation innerhalb des TNM-Systems dar und beschreibt den Tumorstatus, also das Fehlen oder Vorhandensein eines Residualtumors, nach einer (oftmals chirurgischer) Therapie in Bezug auf seine makroskopische und mikroskopische Ausdehnung [46, 47]. In dieser Klassifikation wird nicht nur der Primärtumor, sondern auch der Residualtumor bei entfernten Metastasen berücksichtigt [47].

Die R0-Kategorie („kein Residualtumor") trifft $\mathrm{zu}$, wenn mit konventionellen diagnostischen Methoden kein Residualtumor gefunden werden kann [47]. Die R1-Kategorie liegt vor, wenn ein Residualtumor durch histologische Untersuchung nachgewiesen wurde, einschließlich mikroskopischer Untersuchung der Resektionsränder des chirurgischen Resektats. Die Untersuchung des Resektionsrandes am Präparat dient hierbei gleichsam als Surrogat für die Analyse des Resektionsrandes im Patienten, welcher für eine morphologische Untersuchung aus verständlichen Gründen zumeist nicht zur Verfügung steht. Die R2-Kategorie wird für Fälle mit makroskopischem Nachweis eines Residualtumors angewendet, der entweder klinisch oder durch pathologisch-anatomische Untersuchung nachgewiesen werden kann [46]. In der RX-Kategorie kann keine eindeutige Aussage über den R-Status gemacht werden (• Tab. 1).

Für die Analyse der Vollständigkeit der Tumorresektion, d. h., für die Bestimmung des R-Status, ist die Sorgfalt des Pathologen, mit der die pathologischanatomische Untersuchung durchgeführt wird, von entscheidender Bedeutung [11, 45]. Eine enge Kooperation zwischen Chirurgie und Pathologie ist erforderlich (• Abb. 1; [47]).

In der Praxis kommt es bei der Anwendung der R-Klassifikation nicht selten zu Fehlern. Beispielsweise wäre die Vergabe einer R1- oder R2-Kategorie bei vorliegender Serosaperforation (z. B. beim Magen- oder Kolorektalkarzinom) nicht korrekt, stattdessen muss dies in der pT-Kategorie der jeweiligen Organtumoren berücksichtigt werden [45, 46]. Der Tumorbefall des Grenzlymphknotens (des apikalen Lymphknotens, d. h. des im Lymphabflussgebiet am weitesten vom Tumor entfernt gelegenen Lymphknotens) darf ebenso nicht als R1 gewertet werden, sofern nicht die Resektionslinie durch die Metastase in diesem Lymphknoten verläuft $[11,45]$. Auch der Nachweis einer intra- oder peritumoralen Lymphgefäß-, Venenoder Perineuralinvasion (L1, V1, Pn1) beeinflusst die R-Klassifikation nicht. Bei Vorhandensein von Tumorzellen in Venen oder Lymphgefäßen am Resektionsrand wird dann R1 klassifiziert, wenn Tumorzellen an der Gefäßwand anhaften oder aus dem angrenzenden
Stroma in das Gefäß infiltrieren. Das Vorhandensein von Tumorzellen im Lumen ohne Gefäßwandkontakt darf nicht als R1 gewertet werden [11, 45].

Ein bedeutsamer, klinisch häufig unterschätzter Zusammenhang besteht zwischen der Tumorausbreitung an der Invasionsfront und dem R-Status, wie in $-A b b .2$ schematisch dargestellt: Bei identischem Sicherheitsabstand liegt für Karzinome, die in kompakten glandulären Verbänden wachsen (linke Hälfte), tatsächlich eine R0-Situation vor, während bei Karzinomen mit wenig kohäsivem Wachstum und starker Tumorzelldissoziation, wie etwa dem diffusen Magen- oder dem Pankreaskarzinom, ein R0-Status auch bei fehlendem Tumorzellnachweis am chirurgischen Resektionsrand nicht mit letzter Sicherheit garantiert werden kann (rechte Hälfte; [40]).

\section{Pathologisch-anatomische Aufarbeitung}

\section{Chirurgisch-onkologisches Resektat}

Grundsätzlich ist anzuführen, welche chirurgischen Resektionsränder tumorbefallen oder tumorfrei sind. Neben dem oralen und dem aboralen Resektionsrand ist auch der laterale/zirkumferenzielle Resektionsrand („,circumferential resection margin“, CRM) zu beachten. Diesem kommt speziell beim Ösophagusund Rektumkarzinom, aber auch beim proximalen Magenkarzinom besondere Bedeutung zu [44]. Der CRM wird definiert als der äußere Resektionsrand der adventiziellen Weichteilmanschette, wel- 


\section{Leitthema}

\begin{tabular}{|c|c|}
\hline RX & Vorhandensein von Residualtumor kann nicht beurteilt werden \\
\hline $\mathrm{R} 0>1 \mathrm{~mm}$ & $\begin{array}{l}\text { Kein Residualtumor, minimaler Abstand zwischen Tumor und Resektionsrand } \\
>1 \mathrm{~mm}\end{array}$ \\
\hline $\mathrm{R} 0<1 \mathrm{~mm}$ & $\begin{array}{l}\text { Kein Residualtumor, minimaler Abstand zwischen Tumor und Resektionsrand } \\
1 \mathrm{~mm} \text { oder weniger }\end{array}$ \\
\hline R1-dir & $\begin{array}{l}\text { Mikroskopischer Residualtumor, Tumor direkt am Resektionsrand (Tumor durch- } \\
\text { schnitten) }\end{array}$ \\
\hline R2a & Lokaler makroskopischer Residualtumor \\
\hline $\mathrm{R} 2 \mathrm{~b}$ & Makroskopischer Residualtumor als Fernmetastase(n) \\
\hline $\mathrm{R} 2 \mathrm{c}$ & Makroskopischer Residualtumor in beiden Lokalisationen \\
\hline
\end{tabular}

cher den Tumorausläufern am nächsten ist.

Von entscheidender Bedeutung sind die makroskopische Begutachtung und die sachgerechte Einbettung des Resektionsrandes. So sollte der CRM vom Pathologen (am idealerweise nicht eröffneten Präparat) im Bereich des Tumors zuerst farbmarkiert und danach vollständig unter Mitnahme des Fettgewebes und der darin enthaltenen Lymphknoten zusammen mit dem Tumor eingebettet werden (siehe Abb. 3 am Beispiel eines Ösophagusresektats).

Der minimale Abstand zwischen Tumorrand und Resektionsrand ist auszumessen und (in Millimetern) im Befund anzuführen [44]. Die prognostische Bedeutung der Größe dieses Abstands wurde erstmals für das Rektumkarzinom beschrieben, ihr Stellenwert ist aber auch bei anderen Tumorentitäten von großer Bedeutung [24, 46]. Der R-Status darf nicht mit der CRM-Kategorie verwechselt werden [44]. Folgende drei Situationen können unterschieden werden [44, 46]:

1. CRM-positiv/R1: Tumor direkt am Resektionsrand nachweisbar.

2. CRM-positiv/R0: minimaler Abstand zwischen Tumor und zirkumferenziellem Resektionsrand $\leq 1 \mathrm{~mm}$ aber $>0 \mathrm{~mm}$.

3. CRM-negativ/R0: minimaler Abstand zwischen Tumor und zirkumferenziellem Resektionsrand $>1 \mathrm{~mm}$.

Wichtig ist die Beurteilung des CRM besonders auch nach neoadjuvanter Therapie. Dabei werden nur vitale Tumorzellen berücksichtigt, nicht $\mathrm{z}$. B. azelluläre Schleimseen [44, 45].

\section{Endoskopische Resektate}

Für die pathologische Aufarbeitung und Beurteilung lokaler Tumorexzisate (ohne Entfernung der regionären Lymphknoten) gelten ähnliche Prinzipien. Endoskopische Mukosaresektion (EMR), endoskopische Submukosadissektion (ESD) und umschriebene Vollwandexzision, z. B. im Rahmen der transanalen endoskopischen Mikrochirurgie (TEM) ermöglichen die kurative Entfernung von Frühkarzinomen im Gastrointestinaltrakt, wenn deren Lymphknotenmetastasierungsrisiko vernachlässigbar ist [29].

Für die akkurate histopathologische Untersuchung des Resektats ist eine Abtragung in einem Stück (en bloc) anzustreben, denn eine Abtragung in mehreren Stücken kann nicht nur zu inkompletter Entfernung der Läsion führen, sondern auch die Beurteilung der Abtragungsränder für den Pathologen nahezu unmöglich machen [22, 48].

Die Resektate sollen auf Kork (oder ähnlichem Material) aufgespannt und fixiert werden (die Schleimhautoberfläche nach oben). Dieses erleichtert die Möglichkeit der separaten Aufarbeitung und Markierung der zirkumferenziellen und basalen Resektionsränder, sodass eine Entfernung im Gesunden, die Abstände zu den Resektionsrändern und die Infiltrationstiefe exakt bestimmt werden können [22].

Der Pathologe muss in seinem Befund auf folgende Punkte eingehen ([12, 22]; schematische Darstellung in - Abb.4):

- R-Status: horizontaler (seitlicher) und vertikaler (basaler) Resektionsrand;
- Infiltrationstiefe einschließlich pTKategorie;

- histologischer Tumortyp;

- Differenzierung (Grading);

- Infiltration von Lymph- und Blutgefäßen und/oder Perineuralscheiden.

\section{Schnellschnittuntersuchung}

Wenn intraoperativ der Verdacht besteht, dass Tumorgewebe sehr nahe an den Resektionsrand heranreicht, kann eine Schnellschnittuntersuchung von der verdächtigen Stelle am Resektionsrand veranlasst werden [27]. Je nach Art des Gewebes wird der Pathologe eine Farbmarkierung der Resektionsfläche durchführen und diese histologisch untersuchen. Besonders bei Mammakarzinomen hat sich die Abklatschzytologie als zuverlässige, schnelle und gewebsschonende Technik zur intraoperativen Untersuchung in der Hand eines erfahrenen Pathologen bewährt [7].

\section{Ösophaguskarzinom}

Im Ösophagus treten Plattenepithelund Adenokarzinome etwa gleich häufig auf [46]. Eine anatomisch-pathologische Sonderstellung nehmen Karzinome des gastroösophagealen Übergangs ein. In der aktuellen 8. Auflage der TNM-Klassifikation [4] werden diese als Tumoren definiert, deren Epizentrum in einem Abstand von $2 \mathrm{~cm}$ zum gastroösophagealen Übergang gelegen ist und die auch in den Ösophagus reichen. Entsprechend werden sie wie Tumoren des Ösophagus klassifiziert [4].

Während bei Plattenepithelkarzinomen des mittleren und proximalen Drittels oftmals eine Schnellschnittuntersuchung zur Beurteilung des oralen Resektionsrandes erforderlich sein kann, spielt beim Adenokarzinom des Ösophagus eher der aborale Resektionsrand für den Schnellschnitt eine Rolle [12]. Der zirkumferenzielle Resektionsrand wird unter Schnellschnittbedingungen zumeist nicht berücksichtigt, speziell nach neoadjuvanter Therapie ist dieses gleichsam unmöglich. Bei schwer orientierbaren Resektaten können spezielle Fadenmarkierungen des oralen und aboralen Randes bzw. der Arterienstümpfe 
bei der radikalen Lymphadenektomie (Grenzlymphknoten) für den Pathologen hilfreich sein [12].

In der R0-Situation muss neben dem oralen und aboralen auch der der zirkumferenzielle Resektionsrand tumorfrei sein (unter Angabe des minimalen Abstandes in Millimeter), dieses gilt auch für die Karzinome des gastroösophagealen Übergangs [21]. Derzeit existieren verschiedene Definitionen für eine CRMPositivität beim Ösophaguskarzinom. Während das College of American Pathologists den Tumorzellnachweis direkt am Resektionsrand, also Tumorzellen „on ink“, als CRM-positiv definiert [42], verwendet das Royal College of Pathologists analog zum Rektumkarzinom einen minimalen Tumorabstand zum CRM von weniger als $1 \mathrm{~mm}$ [34].

\section{I) Zur besseren Prognoseab- schätzung wird eine 3-teilige CRM-Kategorie empfohlen}

In einer aktuellen Studie zeigte sich, dass ein Cut-offvon $500 \mu \mathrm{m}$ Mindesttumorabstand zum CRM prognostisch bedeutsam ist [17]. Die Autoren empfehlen daher die Anwendung einer 3-teiligen CRM-Kategorie $(\mathrm{CRM}>500 \mu \mathrm{m}, \mathrm{CRM} \leq 500 \mu \mathrm{m}$, $\mathrm{CRM}=0 \mu \mathrm{m})$ zur besseren Prognoseabschätzung [17]. Im Allgemeinen stellt die R0-Resektion den wichtigsten Prognosefaktor dar mit einem 5-Jahres-Überleben $>50 \%$ (ohne Vorbehandlung) vs. $0 \%$ bei R1- oder R2-Resektion [20]. Ein wichtiger Punkt hinsichtlich einer eventuellen Nachresektion oder Nachbestrahlung ist zudem die Lokalisation des Residualtumors am Präparat, die vom Pathologen im Befund anzugeben ist [12].

\section{Magenkarzinom}

Adenokarzinome des Magens gehören weltweit zu den häufigsten tumorbedingten Todesursachen [21, 30]. Trotz potenziell kurativer Resektion zeigen auch Patienten in frühen Stadien häufig bereits ein schlechtes 5-Jahres-Überleben mit einem hohen Risiko für Lokalrezidive und lymphogene oder Fernmetastasierung. Daher stellen Adenokarzinome des Magens eine große Herausforderung für alle Disziplinen dar [21].

Der Ausbreitungstyp des Magenkarzinoms (nach Laurén: intestinal oder diffus) bestimmt die Therapie und operative Strategie (Resektionsausmaß) und insbesondere den einzuhaltenden Sicherheitsabstand zum proximalen Resektionsrand. Basierend auf Studien von Hermanek et al. $[12,13]$ werden in der aktuell gültigen deutschen S3-Leitlinie zum Magenkarzinom [21] unterschiedliche Sicherheitsabstände für den intestinalen oder diffusen Typ empfohlen: Während bei intestinalen Magenkarzinomen ein Sicherheitsabstand nach proximal von $4-5 \mathrm{~cm}$ (entsprechend $2-3 \mathrm{~cm}$ am frischen Präparat ohne Zug und Spannung) als ausreichend angesehen wird, soll bei diffusen Magenkarzinomen mit diskontinuierlicher Ausbreitung in der Magenwand ein Sicherheitsabstand von $5-8 \mathrm{~cm}$ (entsprechend $>5 \mathrm{~cm}$ am frischen Präparat ohne Zug und Spannung) angestrebt werden [12, 13, 21]. Folglich kommt für Patienten mit Karzinomen des unteren Magendrittels eine subtotale distale Magenresektion infrage, während bei Karzinomen des oberen und mittleren Drittels in den meisten Fällen eine Gastrektomie erforderlich ist [21].

\section{) Die prognostische Bedeutung des proximalen Resektionsrandes ist umstritten}

Seit den 1980er-Jahren haben viele Studien die prognostische Bedeutung des proximalen Resektionsrandes bei proximal und distal gelegenen Magenkarzinomen untersucht [31]. Diese Studien kamen zu unterschiedlichen Ergebnissen, was die prognostische Signifikanz des proximalen Resektionsrandes betrifft [31]. Schwierigkeiten bestehen vor allem in der Interpretation und Uneinheitlichkeit der berichteten makroskopischen und mikroskopischen Sicherheitsabstände [31]. Zudem ist die prognostische Aussagekraft in einer R1-Situation stark vom Tumorstadium abhängig. Während in frühen Stadien eine R1-Resektion mit schlechtem Überleben assoziiert ist, verliert in späten Stadien ein positiver Resektionsrand seine unabhängi-
Chirurg DOI 10.1007/s00104-017-0448-6 ( ) Der/die Autor(en) 2017. Dieser Artikel ist eine Open-Access-Publikation.

M. J. Pollheimer · C. Langner

Pathologie der R1Klassifikation in der viszeralonkologischen Chirurgie

\section{Zusammenfassung}

Die Residualtumor(R-)Klassifikation beschreibt das Fehlen oder Vorhandensein von Tumorgewebe nach erfolgter Therapie. Die R-Kategorie eines chirurgischen Resektats spiegelt den Therapieeffekt wider und ist von entscheidender Bedeutung für das weitere therapeutische Vorgehen und das Patientenüberleben. Für die Bestimmung der R-Kategorie ist eine sorgfältige Analyse aller Resektionsflächen, einschließlich des zirkumferenziellen Resektatrandes, durch den Pathologen notwendig.

Schlüsselwörter

TNM-Klassifikation · Residualtumor ·

Resektionsrand · Prognose · Fernmetastasen

\section{Pathology of the R1 classification in visceral cancer surgery}

\section{Abstract}

The completeness of tumor removal is described in the residual tumor classification (R classification). The $R$ category of a surgical specimen reflects the effects of treatment, influences further treatment decisions and is associated with patient survival. Thorough pathological examination of all resection planes, including the circumferential margin, is necessary for accurate classification.

\section{Keywords}

TNM classification - Residual tumor .

Resection margin · Prognosis · Neoplasm metastasis

ge prognostische Bedeutung und ist ganz allgemein ein Marker für aggressives Tumorverhalten und schlechtes Patientenüberleben [31]. Somit bleibt die prognostische Bedeutung des optimalen Abstandes zum proximalen Resektionsrand umstritten.

Für den distalen Resektionsrand gibt es bis jetzt nur wenige Studien zum chirurgischen Management [31] und die Da- 


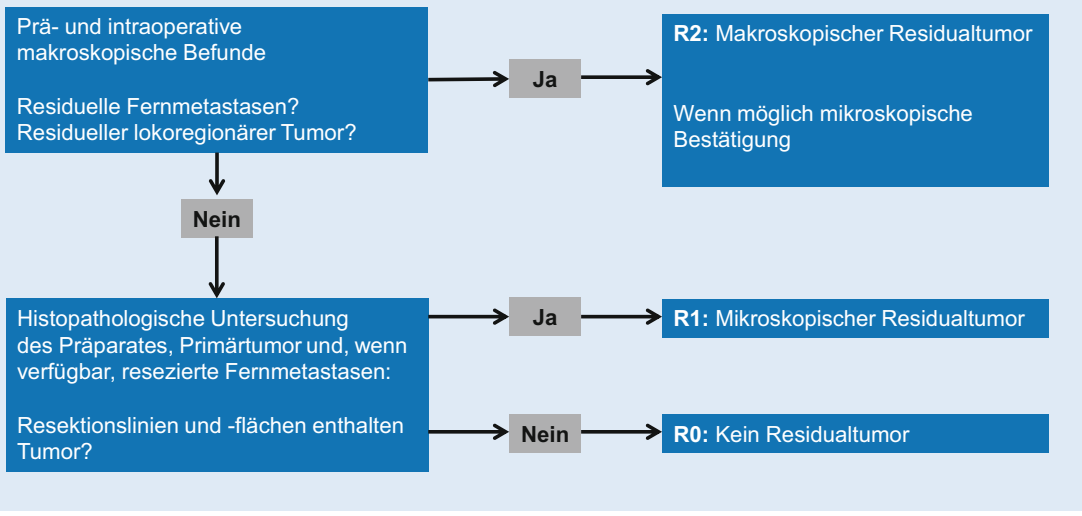

Abb. 1 ム Kooperation zwischen Chirurgie und Pathologie bei der Bestimmung des Residualtumor(R)-Status

tenlage ist nicht eindeutig [16]. Die prognostische Bedeutung des distalen Resektionsrandes muss in zukünftigen prospektiven Studien geklärt werden.

\section{Pankreaskarzinom}

Bei den malignen Tumoren des exokrinen Pankreas ist das duktale Adenokarzinom mit bis zu $90 \%$ der bei weitem häufigste Tumortyp und stellt bei Männern die neunthäufigste und bei Frauen die siebthäufigste maligne Erkrankung überhaupt dar [18]. Anatomisch ist das duktale Adenokarzinom am häufigsten im Kaput (60-70\%), seltener im Korpus (5-15 \%) oder in der Kauda des Pankreas (10-15\%) lokalisiert [3].

Der Tumor hat eine schlechte Prognose mit 5-Jahres-Überlebensraten von unter $10 \%$, was wesentlich durch späte klinische Präsentation und Diagnose sowie schlechtes Therapieansprechen begründet ist [1]. Die wichtigsten Prognosefaktoren sind das Vorhandensein von Lymphknotenmetastasen, Tumordifferenzierung und Perineuralscheideninvasion [35, 40].

In der aktuellen S3-Leitline für das Pankreaskarzinom wird die intraoperative Schnellschnittuntersuchung von Gallengang- und Pankreasschnittrand empfohlen [23]. Dieses gilt nicht für den zirkumferenziellen Resektionsrand. Dieser soll nach Formalinfixierung im Detail aufgearbeitet werden, wobei eine getrennte Farbmarkierung der Resektionsflächen (nach laterokaudal zur Gefäßfurche und nach dorsal/retropankreatisch) empfohlen wird [23]. Der Abstand des Tumors zu den jeweiligen Resektionsrändern soll in Millimetern ausgemessen und im Befund angeführt werden [23].

Bei Karzinomen im Processus uncinatus und im ventralen Pankreaskopf sowie Karzinomen im Kopf-KorpusÜbergangsbereich sind diese Farbmarkierungen von besonderer Wichtigkeit, da diese Karzinome häufig mit engem Bezug zu den zirkumferenziellen Resektionsrändern wachsen und somit häufiger positive Resektionsränder nachzuweisen sind [23].

Über die prognostischen Unterschiede einer R1-Situation der verschiedenen Resektionsränder ist wenig bekannt [37]. Während in einer Studie gezeigt werden konnte, dass eine R1-Situation nur am dorsalen Resektionsrand [9] prognostische Bedeutung für das Patientenüberleben hat, waren in einer anderen Arbeit die Resektionsränder an der Gefäßfurche mit schlechterem Patientenüberleben assoziiert [14]. Die ungleiche prognostische Bedeutung der Lokalisation einer R1-Situation am Pankreaspräparat ist eventuell durch die variierende Dichte an Blut- und Lymphgefäßen sowie Nervenfasern im Bereich der einzelnen Resektionsränder begründet [40].

Die am häufigsten in der Literatur als positiv angegebenen Resektionsränder sind der dorsale und der laterokaudale Resektionsrand zur Gefäßfurche [5, 8, 14, 40], wobei in der Mehrzahl der Studien eine Positivität eines einzelnen Resektionsrandes und nur in etwa einem

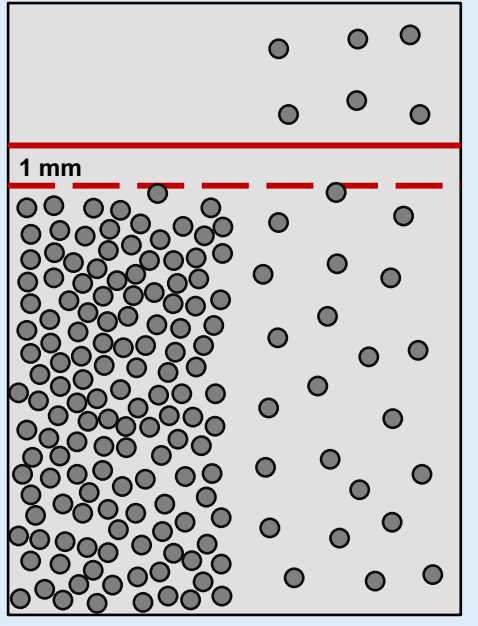

Abb. $2 \Delta$ Die grauen Punkte repräsentieren die Tumorzellen mit kompakten Zellverbänden (linke Hälfte) und wenig kohäsiven Tumorzellen (rechte Hälfte). Die durchgängige Linie zeigt den chirurgischen Resektionsrand. Die gestrichelte Linie kennzeichnet einen Sicherheitsabstand von $1 \mathrm{~mm}$. (Mod. nach [40], mit freundl. Genehmigung von Elsevier). Diese Abbildung ist urheberrechtlich geschützt und unterliegt nicht der Creative Commons Lizenz des Artikels

Drittel zwei bis drei positive Resektionsränder beschrieben werden [37].

Bis dato wird der Einfluss einer R1-Situation nach Pankreatikoduodenektomie auf das Patientenüberleben kontrovers diskutiert [40]. Auffallenderweise unterscheiden sich internationale Studien signifikant in ihren R1-Raten mit großen Schwankungsbreiten von $20-80 \%$ bei nur gering variierenden patienten- und tumorbezogenen Merkmalen [5, 8, 15, 33, 40]. Diese Unterschiede sind auf einen fehlenden Konsens hinsichtlich der Definition des R-Status beim Pankreaskarzinom zurückzuführen.

\section{》) Ein Konsens hinsichtlich der Definition des R-Status beim Pankreaskarzinom fehlt}

In der aktuellen Literatur werden Sicherheitsabstände von 1, 5 oder sogar $10 \mathrm{~mm}$ zum Resektionsrand als R0 angegeben [5, $6,8,10]$. Während in der aktuellen TNMDefinition der R1-Status als mikroskopischer Tumorzellnachweis am CRM festgelegt ist [4], empfiehlt das Royal College of Pathologists einen minimalen Tumor- 

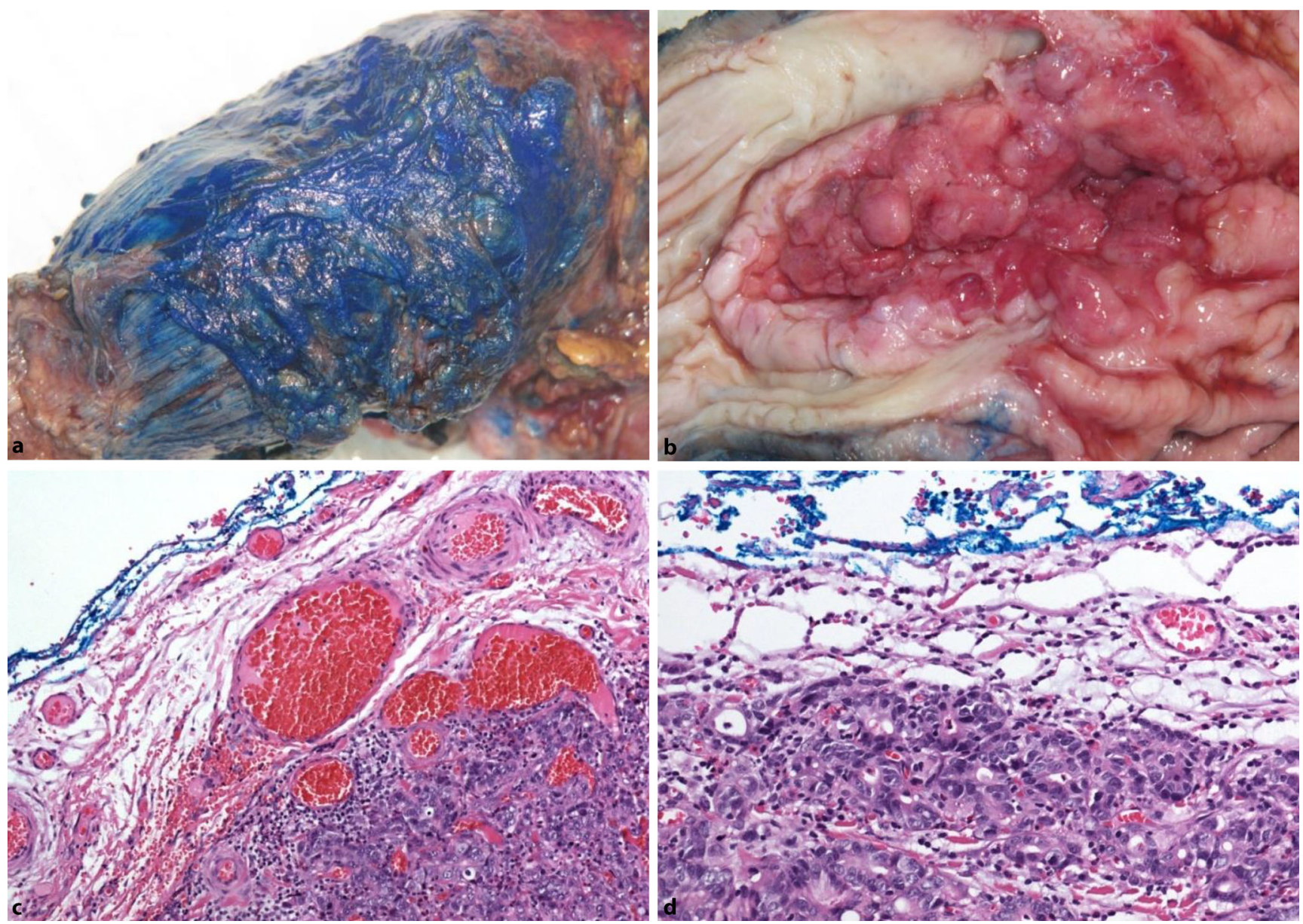

Abb. 3 - Makroskopie (a, b) und Mikroskopie (c, d) eines Ösophagusresektats mit Farbmarkierung der zirkumferenziellen Resektionsfläche

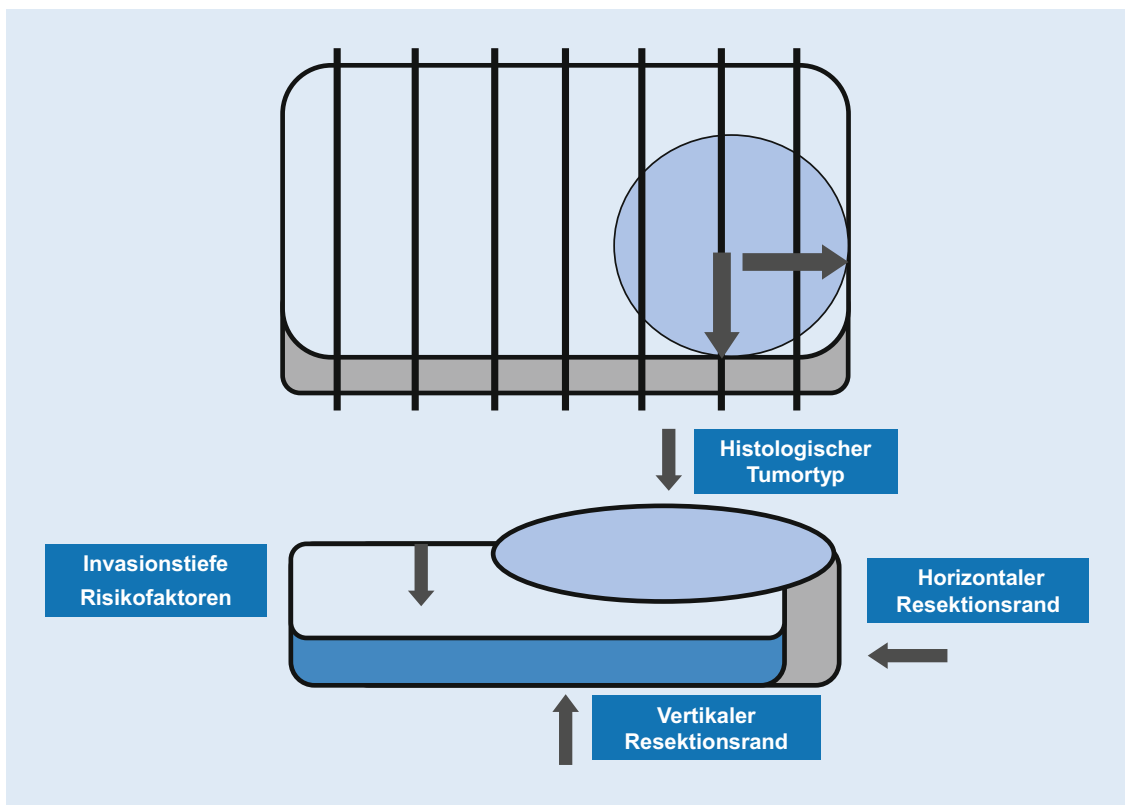

Abb. 4 ॥ Schematische Darstellung der Aufarbeitung eines endoskopischen Tumorexzisats abstand zum CRM von weniger als $1 \mathrm{~mm}$ als R0/CRM-positiv zu klassifizieren [5, 28]. Diese Definition wurde in der aktuellen S3-Leitlinie für das Pankreaskarzinom übernommen [23].

Darüber hinaus hat sich gezeigt, dass eine standardisierte makropathologische Aufarbeitung des Pankreatikoduodenektomiepräparates mit unterschiedlicher Farbmarkierung der chirurgischen und anatomischen Flächen sowie das Ausmaß der Gewebeprobenentnahme die Genauigkeit der Resektionsrandbeurteilung wesentlich beeinflussen [40]. Speziell bedeutet dieses, dass axiale Schnittführung, ausgedehnte Gewebeentnahme und mehrfarbige Randfärbung zu einer signifikant höheren und genaueren R1Rate führen als herkömmliche Dissektionstechniken [40]. 
Tab. 2 Beurteilung der Qualität der Mesorektumexzision

\begin{tabular}{|l|l|l|l|l|}
\hline Qualitätsgrad & Mesorektum & Defekte & Coning & Resektionsrand \\
\hline Grad 1 (komplett) & Intakt, glatt & $<5 \mathrm{~mm}$ & Nein & Glatt, regelmäßig \\
\hline $\begin{array}{l}\text { Grad 2 } \\
\text { (nahezu komplett) }\end{array}$ & $\begin{array}{l}\text { Mäßige Menge, } \\
\text { unregelmäßig }\end{array}$ & $\begin{array}{l}\text { Tunica mus- } \\
\text { cularis propria } \\
\text { nicht sichtbar }\end{array}$ & Mäßig & Unregelmäßig \\
\hline Grad 3 (inkomplett) & Geringe Menge & $\begin{array}{l}\text { Bis zur Tunica } \\
\text { muscularis } \\
\text { propria }\end{array}$ & $\begin{array}{l}\text { Mäßig bis } \\
\text { stark }\end{array}$ & Unregelmäßig \\
\hline
\end{tabular}

\section{Rektumkarzinom}

In den westlichen Industrieländern ist die Inzidenz des kolorektalen Karzinoms im Allgemeinen hoch. Vor allem das Kolonkarzinom steigt in seiner Häufigkeit, während das Rektumkarzinom derzeit eine geringfügig abnehmende Inzidenz aufweist [19].

Kolon- und Rektumkarzinome haben viele Gemeinsamkeiten in Ätiologie und Histologie, unterscheiden sich aber in der präoperativen, der operativen und der adjuvanten Therapiestrategie [39]. Durch die Kombination einer systemischen neoadjuvanten Radiochemotherapie mit einer lokalen Operation gelang in der Therapie des Rektumkarzinoms eine signifikante Reduktion der Lokalrezidivrate und damit eine signifikante Verlängerung der rezidivfreien Überlebenszeit und des Gesamtüberlebens [36].

Die vollständige Entfernung des mesorektalen Gewebes (partielle mesorektale Exzision [PME] bei hoch sitzenden Karzinomen; totale mesorektale Exzision [TME] bei tief sitzenden Karzinomen) mithistologisch tumorfreiem CRM ist für das Rektumkarzinom ein Prognosefaktor von entscheidender Bedeutung [24, $25,36]$.

\section{》) Die Beurteilung der PME/TME-Qualität ist international standardisiert}

Aus pathologischer Sicht ist die Beurteilung der Qualität der mesorektalen Exzision mit einer Farbmarkierung der Resektionsflächen, der genauen makroskopischen Durchsichtung der Schnittflächen und deren Einbettung unter Einbeziehung des Resektatrandes ein integraler Bestandteil einer standardisierten Aufarbeitung von PME- und
TME-Präparaten. Die Beurteilung der Qualität der mesorektalen Exzision wird nach international standardisierten Kriterien (•Tab. 2) durchgeführt. Angaben zum R-Status dürfen in keinem pathologischen Bericht fehlen [36], denn bei inkompletter mesorektaler Exzision ist mit einer signifikant erhöhten Lokalrezidivrate zu rechnen [38].

Für die histologische Beurteilung der Vollständigkeit der mesorektalen Exzision wird der minimale Abstand des Tumors zum nächstgelegenen mesorektalen Resektatrand (unter Angabe der Lokalisation des Randes) am Schnittpräparat ausgemessen und (in Millimetern) im Befund angegeben [36]. Relevant sind sowohl eine Tumorinfiltration per continuitatem, die Beziehung zwischen einer allfälligen Lymphknotenmetastase und dem Resektionsrand oder auch eine intravaskuläre Ausbreitung, da jede Art der Tumorausbreitung an den Resektionsrand mit einem erhöhten Lokalrezidivrisiko einhergeht $[2,36]$. Nach dem EURECCA(European Registration of Cancer Care)-Konsens sollen Abstände von mehr als $1 \mathrm{~mm}$ als R0-Status, Abstände von weniger als $1 \mathrm{~mm}$, ohne dass Tumorzellen direkt am Schnittrand nachweisbar sind, als CRM-positiv/R0 klassifiziert werden [32].

In der aktuellen Literatur werden Lokalrezidivraten von bis $\mathrm{zu} 22 \%$ bei einem Tumorabstand von weniger als $1 \mathrm{~mm}$ zum CRM und Lokalrezidivraten von $5 \%$ bei einem Abstand von mehr als $1 \mathrm{~mm}$ beschrieben [43]. Darüber hinaus wurde gezeigt, dass bei einem Abstand von weniger als $1 \mathrm{~mm}$ mit einer erhöhten Rate von Fernmetastasen zu rechnen ist [25].

Der orale und distale Resektionsrand des Rektumresektats sind meist von untergeordneter Bedeutung, da es operationstechnisch in den meisten Fällen ge- lingt, hier einen ausreichenden Sicherheitsabstand einzuhalten [36]. In der Literatur wird ein Tumorbefall dieser Ränder in nur 1-2\% der Fälle beschrieben $[24,41]$.

\section{Fazit für die Praxis}

\section{- Die R-Klassifikation beschreibt das Fehlen oder Vorhandensein eines Residualtumors nach vorausge- gangener (oftmals chirurgischer) Therapie. \\ - Die R1-Kategorie liegt vor, wenn ein Residualtumor durch histologische Untersuchung nachgewiesen wurde, einschließlich mikroskopischer Un- tersuchung der Resektionsränder des chirurgischen Resektats. Eine enge Kooperation zwischen Chirurgie und Pathologie ist erforderlich. \\ - Neben der Analyse des oralen und aboralen Resektionsrandes liefert auch die systematische Untersu- chung des zirkumferenziellen Re- sektionsrandes prognostische Infor- mationen von eminenter klinischer Bedeutung.}

\section{Korrespondenzadresse}

\section{Univ.-Doz. Dr. C. Langner}

Institut für Pathologie, Medizinische Universität Graz

Auenbruggerplatz 25, 8036 Graz, Österreich cord.langner@medunigraz.at

Open access funding provided by Medical University of Graz.

\section{Einhaltung ethischer Richtlinien}

Interessenkonflikt. M. J. Pollheimer und C. Langner geben an, dass kein Interessenkonflikt besteht.

Dieser Beitrag beinhaltet keine von den Autoren durchgeführten Studien an Menschen oder Tieren.

Open Access Dieser Artikel wird unter der Creative Commons Namensnennung 4.0 International Lizenz (http://creativecommons.org/licenses/by/4.0/deed. de) veröffentlicht, welche die Nutzung, Vervielfältigung, Bearbeitung, Verbreitung und Wiedergabe in jeglichem Medium und Format erlaubt, sofern Sie den/die ursprünglichen Autor(en) und die Quelle ordnungsgemäß nennen, einen Linkzur Creative Commons Lizenz beifügen und angeben, ob Änderungen vorgenommen wurden. 


\section{Literatur}

1. Bickenbach KA, Gonen M, Tang LH et al (2012) Downstaging in pancreatic cancer: a matched analysis of patients resected following systemic treatment of initially locally unresectable disease. Ann Surg Oncol 19:1663-1669

2. Birbeck KF, Macklin CP, Tiffin NJ et al (2002) Rates of circumferential resection margin involvement vary between surgeons and predict outcomes in rectal cancer surgery. Ann Surg 235:449-457

3. Bosman FT, Carneiro T, Hruban RH, Theise ND (Hrsg) (2010) WHO classification of tumours of the digestive system. IARC, Lyon

4. Brierley JD, Gospodarowicz MK, Wittekind C (2017) TNM-Klassifikation maligner Tumoren, 8. Aufl. Springer, Berlin

5. Campbell F, Smith RA, Whelan P et al (2009) Classification of $\mathrm{R} 1$ resections for pancreatic cancer: the prognostic relevance of tumour involvement within $1 \mathrm{~mm}$ of a resection margin. Histopathology 3:277-283

6. Chang DK, Johns AL, Merrett ND et al (2009) Margin clearance and outcome in resected pancreatic cancer. J Clin Oncol 27:2855-2862

7. Esbona K, Li Z, Wilke LG (2012) Intraoperative imprint cytology and frozen section pathology for margin assessment in breast conservation surgery: a systematic review. Ann Surg Oncol 19:3236-3245

8. Esposito I, Kleeff J, Bergmann F et al (2008) Most pancreatic cancer resections are $\mathrm{R} 1$ resections. Ann Surg Oncol 15:1651-1660

9. Gnerlich JL, Luka SR, Deshpande AD et al (2012) Microscopic margins and patterns of treatment failure in resected pancreatic adenocarcinoma. Arch Surg 147:753-760

10. Hartwig W, Hackert T, Hinz U etal (2011) Pancreatic cancer surgery in the new millennium: better prediction of outcome. Ann Surg 254:311-319

11. Hermanek P (1995) pTNM and residual tumor classifications: problems of assessment and prognostic significance. World J Surg 19:184-190

12. Hölscher AH, Fetzner UK (2012) Carcinoma of the esophagus, esophagogastric junction, and stomach. Cooperation between pathology and surgery. Pathologe 33(Suppl 2):246-252

13. Hornig D, Hermanek P, Gall FP (1987) The significance of the extent of proximal margin of clearance in gastric cancer surgery. Scand J Gastroenterol 22(Suppl 133):69-71

14. Jamieson NB, Foulis AK, Oien KA etal (2010) Positive mobilization margins alone do not influence survival following pancreatico-duodenectomy for pancreatic ductal adenocarcinoma. Ann Surg 251:1003-1010

15. Janot MS, Kersting S, Belyaev $O$ et al (2012) Can the new RCP R0/R1 classification predict the clinical outcome in ductal adenocarcinoma of the pancreatic head? Langenbecks Arch Surg 397:917-925

16. Lee CM, Jee YS, Lee JH et al (2014) Length of negative resection margin does not affect local recurrence and survival in the patients with gastric cancer. World J Gastroenterol 20:10518-10524

17. Lee GD, Lee SE, Kim KM et al (2015) New 3-tiered circumferential resection margin criteria in esophageal squamous cell carcinoma. Ann Surg 262:965-971

18. Lüttges J, Stigge C, Pacena M, KlöppelG (2004) Rare ductal adenocarcinoma of the pancreas in patients younger than age 40 years. Cancer 100:173-182

19. Majek O, Gondos A, Jansen L et al (2012) Survival from colorectal cancer in Germany in the early $21 \mathrm{st}$ century. Br JCancer 106:1875-1880
20. Metzger R, Drebber U, Baldus SE et al (2009) Extracapsular lymph node involvement differs between squamous cell and adenocarcinoma of the esophagus. Ann Surg Oncol 16:447-453

21. Moehler M, Al-Batran SE, Andus T et al (2011) German S3-guideline "Diagnosis and treatment of esophagogastric cancer". Z Gastroenterol 49:461-531

22. Munding J, Ziebarth W, Belyaev O, UhIW, Tannapfel A (2013) Histological classification: evaluation of resection specimens of EMR/ESD - response evaluation after neoadjuvant therapy. Zentralbl Chir 138(Suppl 2):e55-e62

23. Munding J, Lüttges J, Esposito I, Tannapfel A (2014) Update of the S3 guidelines for pancreatic cancer. What is new for pathologists? Pathologe 35:509-518

24. Nagtegaal ID, Quirke P (2008) What is the role for the circumferential margin in the modern treatment of rectal cancer? JClin Oncol 26:303-312

25. Nagtegaal ID, van Krieken JH (2002) The role of pathologists in the quality control of diagnosis and treatment of rectal cancer-an overview. Eur J Cancer 38:964-972

26. Nagtegaal ID, Quirke P, Schmoll HJ (2011) Has the new TNM classification for colorectal cancer improved care? Nat Rev Clin Oncol 9:119-123

27. Oberschmid B, Dietrich A, Wittekind C (2012) Frozen sections diagnostics in visceral surgery. Stomach and intestines. Pathologe 33:407-412

28. Pancreatric Section, British Society of Gastroenterology, Pancreatic Society of Great Britain and Ireland, Association of Upper Gastrointestinal Surgeons of Great Britain and Ireland, Royal College of Pathologists, Special Interest Group for Gastro-Intestinal Radiology (2005) Guidelines for the management of patients with pancreatic cancer periampullary and ampullary carcinomas. Gut 54(Suppl 5):v1-v16

29. Pimentel-Nunes $P$, Dinis-Ribeiro $M$, Ponchon $T$ et al (2015) Endoscopic submucosal dissection: European Society of Gastrointestinal Endoscopy (ESGE) guideline. Endoscopy 47:829-854

30. van der Post RS, Vogelaar IP, Carneiro F et al (2015 Hereditary diffuse gastric cancer: updated clinical guidelines with an emphasis on germline $\mathrm{CDH} 1$ mutation carriers. J Med Genet 52:361-374

31. Postlewait LM, Squires MH 3rd, Kooby DA et al (2015) The importance of the proximal resection margin distance for proximal gastric adenocarcinoma: a multi-institutional study of the US Gastric Cancer Collaborative. J Surg Oncol 112:203-207

32. Quirke P, West NP, Nagtegaal ID (2014) EURECCA consensus conference highlights about colorecta cancer clinical management: the pathologists expert review. Virchows Arch 464:129-134

33. Raut CP, Tseng JF, Sun CC et al (2007) Impact of resection status on pattern of failure and surviva after pancreaticoduodenectomy for pancreatic adenocarcinoma. Ann Surg 246:52-60

34. Royal College of Pathologists (2007) Standards and dataset for reporting cancers dataset for the histopathological reporting of oesophageal carcinoma (2nd edition). https://www.rcpath.org/resourceLibrary/ g006oesophagealdatasetfinalfeb07-pdf.html.Zugegriffen: 10.03.2017

35. Schmidt CM, Powell ES, Yiannoutsos CT et al (2004) Pancreaticoduodenectomy: a 20-year experience in 516 patients. Arch Surg 139:718-725

36. Schmitz KJ, Chmelar C, Berg E, Schmid KW (2011) Pathological work-up of rectal cancer following partial/total mesorectal excision. Pathologe 32:321-329

37. Schlitter AM, Esposito I (2010) Definition of microscopic tumor clearance $(\mathrm{r} 0)$ in pancreatic cancer resections. Cancers (Basel) 2:2001-2010

38. Stelzner S, Koehler C, Stelzer J et al (2011) Extended abdominoperineal excision vs. standard abdominoperineal excision in rectal cancer a systematic overview. Int J Colorectal Dis 26:1227-4120

39. Tamas K, Walenkamp AM, de Vries EG et al (2015) Rectal and colon cancer: not just a different anatomic site. Cancer Treat Rev 41:671-679

40. Verbeke CS (2013) Resection margins in pancreatic cancer. Surg Clin North Am 93:647-662

41. Wang Z, Zhou Z, Wang C et al (2005) Microscopic spread of low rectal cancer in regions of the mesorectum: detailed pathological assessment with whole-mount sections. Int J Colorectal Dis 20:231-237

42. Washington K, Berlin J, Branton P et al (Hrsg) (2016) Protocol for the examination of specimens from patients with carcinoma of the ampulla of Vater. College of American Pathologists (CAP). http://www.cap.org/ShowProperty?nodePath=/ UCMCon/Contribution\%20Folders/WebContent/ pdf/cp-ampulla-15protocol-3200.pdf. Zugegriffen: 10.03 .2017

43. Wibe A, Rendedal PR, Svensson E et al (2002) Prognostic significance of the circumferential resection margin following total mesorectal excision for rectal cancer. Br J Surg 89:327-334

44. Wittekind C, Compton C, Quirke P et al (2009) A uniform residual tumor $(\mathrm{R})$ classification: integration of the R classification and the circumferential margin status. Cancer 115:3483-3488

45. Wittekind C (2007) Problems with residual tumor classification, particularly R1. Chirurg 78:785-791

46. Wittekind C (2009) Residual tumor classification in oncology - applications and problems. Zentralbl Chir 134:3-6

47. Wittekind C, Compton C, Brierley J, Sobin LH (Hrsg) (2012) TNM supplement A commentary on uniform use, 4. Aufl. Wiley-Liss, New York

48. Ziachehabi A (2014) Endoskopische Submukosadissektion. Wien Klin Mag 17:8-11 